International Journal of Engineering, Science and Technology

Vol. 3, No. 6, 2011,pp. 44-60
INTERNATIONAL

JOURNAL OF

ENGINEERING,

SCIENCE AND

TECHNOLOGY

www.ijest-ng.com

(C) 2011 MultiCraft Limited. All rights reserved

\title{
Neuro- PI controller based model reference adaptive control for nonlinear systems
}

\author{
R. Prakash ${ }^{1}$, R. Anita ${ }^{2}$ \\ ${ }^{1}$ Department of Electrical and Electronics Engineering, Muthayammal Engineering College, Rasipuram, Tamilnadu, INDIA-636102, \\ prakashragu@yahoo.co.in \\ ${ }^{2}$ Department of Electrical and Electronics Engineering, Institute of Road and Transport Technology, Erode, Tamilnadu, INDIA-638316, \\ anita_irtt@yahoo.co.in
}

\begin{abstract}
The aim of this paper is to design a neural network based intelligent adaptive controller. It consists of an online multilayer back propagation neural network structure along with a conventional Model Reference Adaptive Control (MRAC).The training patterns for the Neural Network (NN) are obtained from the conventional PI controller. In the conventional model reference adaptive control (MRAC) scheme, the controller is designed to realize plant output converges to reference model output based on the plant which is linear. The NN is used to compensate the nonlinearity of the plant that is not taken into consideration in the conventional MRAC. The control input to the plant is given by the sum of the output of conventional MRAC and the output of NN. The proposed Neural Network -based Model Reference Adaptive Controller (NN-MRAC) can significantly improve the system behavior and force the system to follow the reference model and minimize the error between the model and plant output. The effectiveness of the proposal control scheme is demonstrated by simulations.
\end{abstract}

Keywords: Model Reference Adaptive Controller (MRAC), Artificial Neural Network (ANN), Proportional-Integral (PI) controller

DOI: http://dx.doi.org/10.4314/ijest.v3i6.4S

\section{Introduction}

In the adaptive literature, the question of control of nonlinear systems with present-day sophistication and complexities has often been an important research area due to the difficulties in modeling, nonlinearities and uncertainties. Model Reference Adaptive Control (MRAC) is one of the main schemes used in adaptive system. Recently MRAC has received considerable attention, and many new approaches have been applied to practical process (Astrom and Wittenmark, 1995). (Petros A loannou et al, 1996).In the MRAC scheme, the controller is designed to realize plant output converges to reference model output based on assumption that plant can be linearzed. Therefore this scheme is effectively for controlling linear plants with unknown parameters. However, it may not assure for controlling nonlinear plants with unknown structure. In recent years, an Artificial Neural network (ANN) has become very popular in many control applications due to their higher computation rate and ability to handle nonlinear system. Some of the relevant research work including ANN as a part of control scheme is illustrated next. A robust Adaptive control of uncertain nonlinear system using neural network is discussed in (Mofal and Calise, 1997). Various types of NN have been efficiently utilized in identification of nonlinear systems (Chen et al., 1991; (Guchi and Sakai, 1991). A variety of algorithms are utilized to adjust the weight of the NN. In a typical multilayered NN, the weights in the layers can be adjusted as to minimize the output error between the NNs output and the observed output. The back propagation algorithm for efficiently updating the weight is useful in many applications such identification of nonlinear systems. Off-line iterative algorithm can be employed in such care of identification or modeling. However, in the aspect of control, the NN should work in on line manner. In the control system structure, the output of $\mathrm{NN}$ is the control input to the nonlinear controlled system. i.e., there is the 
unknown nonlinear system between the $\mathrm{NN}$ and the output error. In this case, in order to apply any learning rules, we need the derivatives of the system output with respect to the input (Yamada and Yabuta, 1992). (Kawalo, et al 1987) presented a simple structure of NN based feed forward controller which is equivalently an inverse of the controlled system after the NN completes learning of the weights which are adjusted to minimize the feedback error. (Narendra and parthasarathy, 1990) has shown in general indirect approach to nonlinear discrete time neuro-control scheme which consists of identification and adaptive control by using the (Chen, 1990) and (Liu and chen, 1993) that the NN-based adaptive control algorithm can cooperate well with identification of the nonlinear functions to realize a nonlinear adaptive control when the nonlinear adaptive control scheme is feedback linearizable. (Kamalsudan and Ghandakly, 2010) presented a fighter aircraft pitch controller evolved from a dynamic growing RBFNN in parallel with a model reference adaptive controller. The abilities of a neural network for nonlinear approximation and development for nonlinear approximation and the development of a nonlinear adaptive controller based on NNs has been discussed in many works (Calise, et al, 2001).(Arciniegas et al,1997). A Neural Network Internal Model Control (NN- IMC) strategy is investigated in (Totok R. Biyanto et al, 2010) by establishing inverse and forward model based neural network (NN). The use of NNs for identification and control of non linear system has been demonstrated in (Ahmed, 2000) discusses a direct adaptive neural network controller for a class of non linear system.

An online Radial Basis-Function Neural Network (RBFNN) in parallel with a MRAC is discussed in (Kamalasadan and Ghandakly, 2007). (Yujia Zhai et al, 2010) proposed a nonlinear model predictive control (NMPC) method to control the elevation and travel of a three degree of freedom (DOF) laboratory helicopter using successive linearization to approximate the internal model of the system. An adaptive-neuro-fuzzy-based sensorless control of a smart-material actuator is presented in (Sadighi and Kim, 2011). An Adaptive Inverse Model Control System (AIMCS) is designed for the plant, and two Radial Basis Function Neural Networks (RBF) NNs are utilized in the AIMCS discussed in (Yuan Xiaofang et al, 2010). (Xiang-Jie Liu et al, 2004) discussed an Adaptive Inverse Model Control System (AIMCS) and it is designed for the plant, and two RBF NNs are utilized in the AIMCS. A MRAC-based current control scheme of a Permanent magnet (PM) synchronous motor with an improved servo performance is presented in (Cho et al, 2009). (Fadali et al, 2010) presented a robust adaptive control approach using MRAC for autonomous robot systems with random friction. An adaptive output-feedback control scheme is developed for a class of nonlinear Single Input Single Output (SISO) dynamic systems with time delays (Mirkin et al, 2010). A neuro-sliding mode approach based on MRAC is proposed in (Huh and Bien, 2007). In particular, the adaptive tracking control architecture proposed in (Sanner and Slotine, 1992) evaluated a class of continuous-time nonlinear dynamic systems for which an explicit linear parameterization of uncertainty is either unknown or impossible.

The adaptive controller is used in various practical applications have attracted much attention in the field of control engineering. This is due to their promising potential for the tasks of tackling the presence of unknown parameters or unknown variation in plant parameters with better performance than those of constant gain feedback control law. In general, the external load disturbances always exist, although it is bounded. So, the controller without considering the disturbances can not stabilize the closed-loop control system. A solution to this problem is to incorporate dead-zone technique in the adaptive controller. With this approach, the controller will stop updating the control parameters when the identifier error is smaller than some threshold. Thus, it can prevent the estimated parameters from being infinity. However, the regulation error of the system will only be asymptotically bounded if large threshold is used, resulting in undesirable closed-loop performance. All control techniques have their individual characteristics. Hence, combining the merits of the adaptive control with that of the neural network control theories and then designing a new stabilizing controller will have better performance than that based on one control theory

PI controllers are widely used in industrial control systems applications. They have a simple structure and can offer a satisfactory performance over a wide range of operation. Therefore, the majority of adaptation schemes described in the literature for MRAS employ a simple fixed-gain linear PI controller to generate the estimated output. However, due to the continuous variation in the plant parameters and the operating conditions, in addition to the nonlinearities present in the plant, PI controllers may not be able to provide the required performance. Not much attention has been devoted to study other types of adaptation mechanisms used to minimize the error to obtain the estimated output.

In this paper, this point is addressed by presenting a neural network to replace the classical PI controller used in the conventional MRAC scheme. The NN -MRAC scheme is proposed as nonlinear optimizer, which ensures plant output trajectory tracks the reference model output trajectory and tracking error is zero with minimum time as possible. From the designed PI controller training patterns are generated and it is used to train the artificial BPN neural network. The trained neural network connected in parallel with an MRAC and it output the appropriate control signals for achieving the desired response. The control input given by the plant is the sum of the output of adaptive controller and the output of neural network. The neural network is used to compensate the nonlinearity of the plant that is not taken into consideration in the conventional MRAC. The role of model reference adaptive controller is to perform the model matching for the uncertain linearized system to a given reference model. The network weights are adjusted by multilayer back propagation algorithm which is carried out in online. Finally to confirm the effectiveness of proposed method, it is compared with the simulation results of the conventional MRAC.

The paper is organized as follows. Section 2 proposes the structure of an MRAC design. Section 3 describes the PI controllerbased model reference adaptive controller and Section 4 describes the discussion of the proposed scheme. Section 5 analyses the result and discussion of the proposed Neural Network-based Model Reference Adaptive Control (NN-MRAC) scheme and the conclusions are given in section 6. In this paper, the proposed NN-MRAC scheme is designed by using parallel combination of the conventional MRAC system and NN controller. 


\section{Structure of an MRAC design}

The MRAC is one of the major approaches in adaptive control. The desired performance is expressed as a reference model, which gives the wished response to an input signal. The adjustment mechanism changes the parameters of the regulator by minimizing the error between the system output and the reference model.

\subsection{The Plant Model and Reference Model System.}

To consider a Single Input and Single Output (SISO), Linear Time Invariant (LTI) plant with strictly proper transfer function

$G(s)=\frac{y_{P}(s)}{u_{p}(s)}=K_{P} \frac{Z_{p}(s)}{R_{P}(s)}$

where $u_{p}$ is the plant input and $y_{p}$ is the plant output. Also, the reference model is given by

$G_{m}(s)=\frac{y_{m}(s)}{r(s)}=K_{m} \frac{Z_{m}(s)}{R_{m}(s)}$

where $r$ and $y_{m}$ are the model's input and output. Define the output error as

$e=y_{p}-y_{m}$

Now the objective is to design the control input $U_{m r}$ such that the output error, $e$ goes to zero asymptotically for arbitrary initial condition, where the reference signal $r(t)$ is piecewise continuous and uniformly bounded. The plant and reference model satisfy the following assumptions:

Assumptions:

1. $\mathrm{Z}_{\mathrm{p}}(\mathrm{s})$ is a monic Hurwitz polynomial of degree $\mathrm{m}_{\mathrm{p}}$

2. An upper bound $n$ of degree $n_{p}$ of $R_{p}(S)$

3. The relative degree $\mathrm{n}^{*}=\mathrm{n}_{\mathrm{p}}-\mathrm{m}_{\mathrm{p}}$ of $G(s)$

4. The sign of the high frequency gain $K_{p}$ are known

5. $Z_{\mathrm{m}}(\mathrm{s}), \mathrm{R}_{\mathrm{m}}(\mathrm{s})$ are monic Hurwitz polynomials of degree $\mathrm{q}_{\mathrm{m}}, \mathrm{p}_{\mathrm{m}}$ respectively, where $\mathrm{p}_{\mathrm{m}} \leq \mathrm{n}$

6. The relative degree $\mathrm{n}_{\mathrm{m}}{ }^{*}=\mathrm{p}_{\mathrm{m}-} \mathrm{q}_{\mathrm{m}}$ of $G_{m}(s)$ is the same as that of $G(S)$, i.e., $\mathrm{n}_{\mathrm{m}}{ }^{*}=\mathrm{n}^{*}$

\subsection{Relative Degree $n=1$}

As in Ref [1] the following input and output filters are used,

$\dot{\omega}_{1}=F \omega_{1}+g u_{p}$

$\dot{\omega}_{2}=F \omega_{2}+g y_{p}$

where $F$ is an $(n-1) *(n-1)$ stable matrix such that $\operatorname{det}(S I-F)$ is a Hurwitz polynomial whose roots include the zeros of the reference model and that $(F, g)$ is a controllable pair. It is defined as the "regressor" vector

$\omega=\left[\omega_{1}^{T}, \omega_{2}^{T}, y_{p}, r\right]^{T}$

In the standard adaptive control scheme, the control $U_{m r}$ is structured as

$U_{m r}=\theta^{T} \omega$

where $\theta=\left[\theta_{1}, \theta_{2}, \theta_{3}, C_{0}\right]^{T}$ is a vector of adjustable parameters, and is considered as an estimate of a vector of unknown system parameters $\theta^{*}$.

The dynamic of tracking error

$e=G_{m}(s) p^{*} \widetilde{\theta}^{T} \omega$

where $P^{*}=\frac{K_{p}}{K_{m}}$ and $\widetilde{\theta}=\theta(t)-\theta^{*} \quad$ represents parameter error. Now in this case, since the transfer function between the parameter error $\widetilde{\theta}$ and the tracking error $e$ is strictly positive real (SPR) [1], the adaptation rule for the controller gain $\theta$ is given by

$\dot{\theta}=-\Gamma e_{1} \omega \operatorname{sgn}\left(p^{*}\right)$

where $\Gamma$ is a positive gain. 


\subsection{Relative Degree $n=2$}

In the standard adaptive control scheme, the control $U_{m r}$ is structured as

$U_{m r}=\theta^{T} \omega+\dot{\theta}^{T} \Phi=\theta^{T} \omega-\theta^{T} \Gamma \phi e_{1} \operatorname{sgn}\left(K_{p} / K_{m}\right)$

where $\theta=\left[\theta_{1}, \theta_{2}, \theta_{3}, C_{0}\right]^{T}$ is a vector of adjustable parameters, and is considered as an estimate of a vector of unknown system parameters $\theta^{*}$.

The dynamic of tracking error is

$e=G_{m}(s)\left(s+p_{0}\right) p^{*} \widetilde{\theta}^{T} \phi$

where $P^{*}=\frac{K_{p}}{K_{m}}$ and $\tilde{\theta}=\theta(t)-\theta^{*}$ represent the parameter error. $G_{m}(s)\left(s+p_{0}\right)$ is strictly proper and Strictly Positive Real(SPR). Now in this case, since the transfer function between the parameter error $\widetilde{\theta}$ and the tracking error $e$ is SPR, [1] and the adaptation rule for the controller gain $\theta$ is given

$\dot{\theta}=\Gamma \phi e_{1} \operatorname{sgn}\left(K_{p} / K_{m}\right)$

where $e_{I=} y_{p} y_{m}$ and $\Gamma$ is a positive gain.

The adaptive laws and control schemes developed are based on a plant model that is free from disturbances, noise and unmodelled dynamics. These schemes are to be implemented on actual plants that most likely to deviate from the plant models on which their design is based. An actual plant may be infinite in dimensions, nonlinear and its measured input and output may be corrupted by noise and external disturbances. It is shown by using conventional MRAC that adaptive scheme is designed for a disturbance free plant model and may go unstable in the presence of small disturbances.

\section{PI Controller-based Model Reference Adaptive Controller}

The PI algorithm remains the most popular approach for industrial process control, despite continual advances in control theory. This is because the PI algorithm has a simple structure which is conceptually easy to understand and implement in practice but also the algorithm provides adequate performance in the vast majority of applications. A PI controller may be considered as an extreme form of a phase lag compensator. The transfer function of PI Controller is generally written in the "Parallel form" given by (12) or the "ideal form" given by (13).

$G_{P I}(S)=\frac{U_{p i}(S)}{E(S)}=K_{P}+\frac{K_{i}}{S}$

$=K_{P}\left(1+\frac{1}{T_{i}}\right)$

where $\mathrm{U}_{\mathrm{pi}}(S)$ is the control signal acting on the error signal $E(s), K_{p}$ is the proportional gain, $K_{i}$ is the integral gain and $T_{i}$ is the integral time constant. The "two term" functionalities are highlighted by the following.

- The proportional term-providing an overall control action proportional to the error signal through the all - pass gain factor.

- The integral term - reducing steady state errors through low - frequency compensation by an integrator

The disturbance and nonlinear component are added to the plant input of the conventional model reference adaptive controller, in this case the tracking error has not reaches to zero and the plant output is not tracked with the reference model plant output. The conventional MRAC fails completely under the action of the external disturbance and nonlinearities, where a degradation in the performance due to overshoot is observed. The disturbance is considered as a random noise signal. To improve the system performance, the PI controller-based Model Reference Adaptive Control scheme (PI-MRAC) is proposed. In this scheme, the controller is designed by using parallel combination of conventional MRAC system and PI controller. The Block diagram of PIMRAC scheme is shown in Fig.1. The control input $U_{m p}$ of the plant is given by the following equation,

$U_{m p}=U_{m r}+U_{p i}$

where $U_{m r}$ is the output of the adaptive controller and $U_{p i}$ is the output of the PI controller.

$U_{m r}=\theta^{T} \omega$

where $\theta$ is the update law vector, and $\omega$ is the parameter vector. 


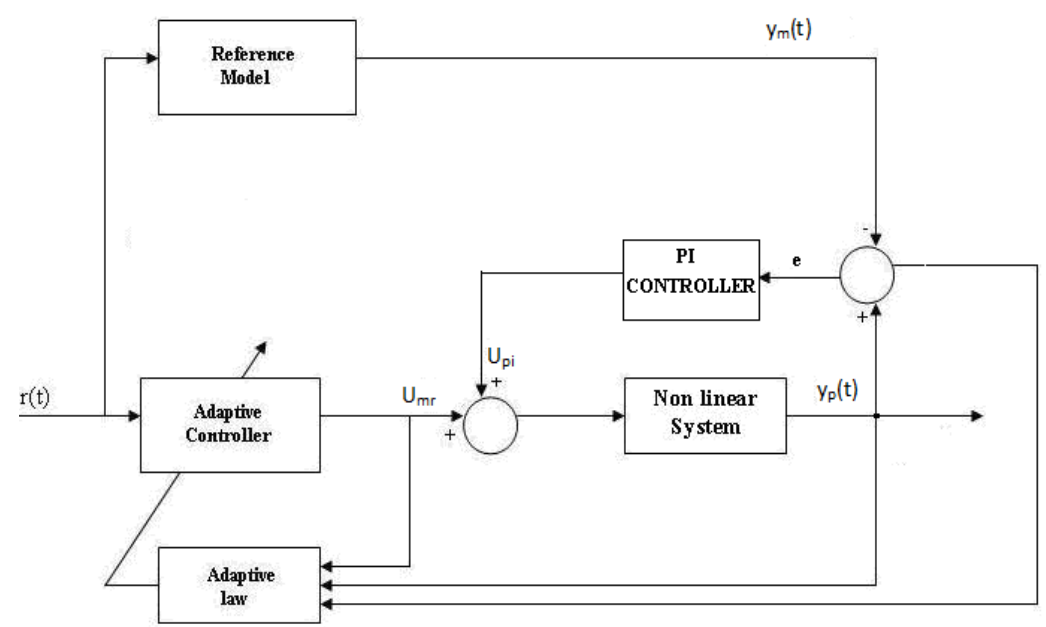

Fig.1 Block diagram of PI-MRAC scheme

The input of the PI controller is the error, in which the error is the difference between the plant output $y_{p}(t)$ and the reference model output $y_{m}(t)$. The PI controller gains can be selected as high as possible, but are limited by the noise (Vas, 1998). In this paper PI gains are obtained by trial and error method. In this case also, the disturbance (random noise signal) and nonlinear component is added to the input of the plant. The PI-MRAC scheme improves the system performance comparing to the conventional MRAC scheme. However due to the continuous variation in the system parameters and the operating conditions, in addition to the nonlinearities present in the system, PI-MRAC scheme may not be able to provide the required performance.

\section{Neural Network-based Model Reference Adaptive Controller}

To make the system more quickly and efficiently adaptable than conventional MRAC system and PI-MRAC scheme, a new idea is proposed and implemented. The new idea which is proposed in this paper is the Neural Network-based Model Reference Adaptive Controller (NN-MRAC). In this scheme, the controller is designed by using parallel combination of conventional MRAC system and NN controller. The training patterns of NN are extracted from the input and output of PI controller of designed PI -MRAC scheme. The block diagram of proposed NN-MRAC scheme is shown in Fig. 2

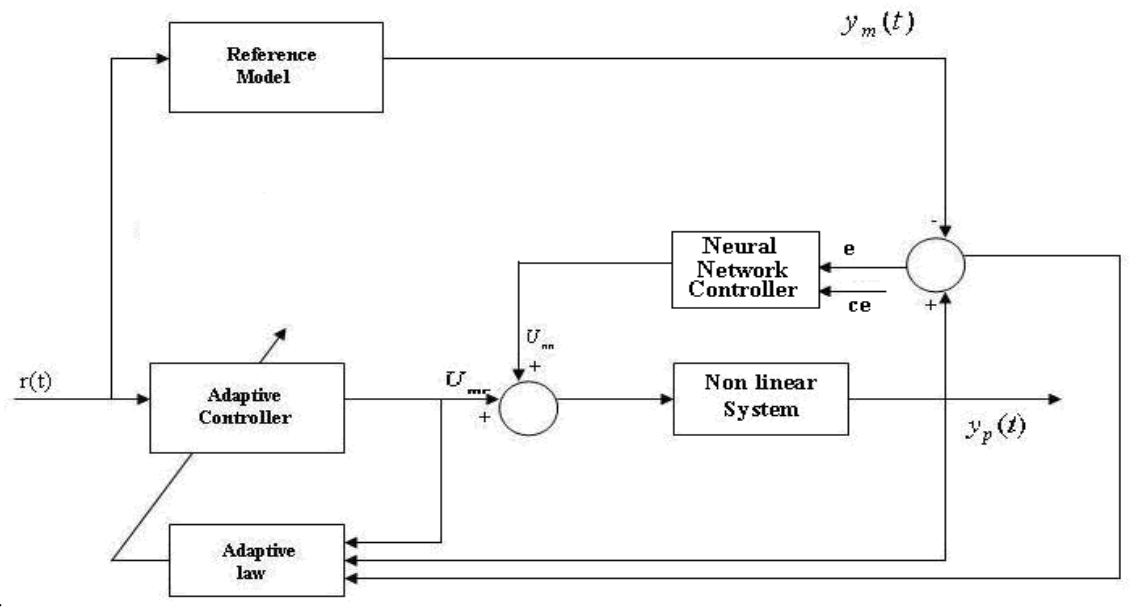

Fig. 2 Block diagram of the proposed MRAC scheme

This scheme is restricted to a case of Single Input Single Output (SISO) control, noting that the extension to Multiple Input Multiple Output (MIMO) is possible. To keep the plant output $y_{p}$ converges to the reference model output $y_{m}$, it is synthesize the control input $U_{m n}$ by the following equation,

$U_{m n}=U_{m r}+U_{n n}$

where $U_{m r}$ is the output of the adaptive controller

$U_{m r}=\theta^{T} \omega$ 


$$
\begin{aligned}
\theta & =\left[\theta_{1}, \theta_{2}, \theta_{3}, C_{0}\right]^{T} \\
\omega & =\left[\omega_{1}, \omega_{2}, y_{p}, r\right]^{T}
\end{aligned}
$$

where $\theta$ is the update law vector, and $\omega$ is the parameter vector.

Stability of the system and adaptability are then achieved by an adaptive control law $U_{m r}$ tracking the system output to a suitable reference model output, such as that the error $\mathrm{e}=\mathrm{y}_{\mathrm{p}}-\mathrm{y}_{\mathrm{m}}=0$ asymptotically. The NN provides an adaptive control for better system performance and solution for controlling nonlinear processes.

The ANN controllers designed in most of the work use a complex network structure for the controller. The aim of this work is to design a simple ANN controller with as low neurons as possible while improving the performance of the controller. The inputs of the NN are the error and change in error. Here the multilayer back propagation NN is used in the proposed method. The multilayer back propagation network is especially useful for this purpose, because of its inherent nonlinear mapping capabilities, which can deal effectively for real-time online computer control. The NN of the proposed method has three layers: an input layer with 2 neurons, a hidden layer with 2 neurons and an output layer with one neuron.

Let $x_{i}$ be the input to the $\mathrm{i}^{\text {th }}$ node in the input layer, $z_{j}$ be the input to the $\mathrm{j}^{\text {th }}$ node in the hidden layer, $y$ be the input to the node in the output layers. Furthermore $V_{i j}$ be the weight between the input layer and hidden layer, $W_{j 1}$ is the weight between the hidden layer and the output layer. The relations between inputs and output of $\mathrm{NN}$ are expressed as,

$$
\begin{aligned}
& Z_{-i n j}=V_{o j}+\sum_{i=1}^{n} x_{i} V_{i j} \\
& z_{j}=F\left(Z_{-i n j}\right) \\
& Y_{-i n}=W_{01}+\sum_{j=1}^{P} z_{j} W_{j 1} \\
& y=F\left(Y_{-i n}\right)
\end{aligned}
$$

where $F($.$) is the activation function.$

Sigmoid function is chosen for the activation function as follows

$$
F(x)=\frac{2 a}{1+\exp (-\mu x)}-a
$$

where $\mu>0, a$ is a specified constant such as that $a \leq 0$, and $F(x)$ satisfies $-a<F(x)<a$

The aim of training is to minimize the sum of square error energy function given by $E(k)=\frac{1}{2}\left[t_{k}-y_{k}\right]^{2}$

The weight are updated by using

$$
\begin{gathered}
\Delta W_{j 1}=-\eta \frac{\partial E}{\partial W_{j 1}}=-\eta\left(t_{k}-y_{k}\right) F^{1}\left(Y_{-i n}\right) z_{j} \\
\Delta W_{01}=-\eta \frac{\partial E}{\partial W_{01}}=-\eta\left(t_{k}-y_{k}\right) F^{1}\left(Y_{-i n}\right) \\
\Delta V_{i j}=-\eta \frac{\partial E}{\partial V_{i j}}=-\eta F^{1}\left(Z_{-i n j}\right) x_{i} \sum_{k} \delta_{k} W_{j 1} \\
\Delta V_{0 j}=-\eta \frac{\partial E}{\partial V_{0 j}}=-\eta F^{1}\left(Z_{-i n j}\right) \sum_{k} \delta_{k} W_{j 1}
\end{gathered}
$$

where $\eta$ is the learning role

$$
\begin{aligned}
& \frac{\partial F\left(Y_{-i n}\right)}{\partial\left(Y_{-i n}\right)}=\frac{\mu}{2 a}\left[\left(a-F\left(Y_{-i n}\right)\right)\left(a+F\left(Y_{-i n}\right)\right)\right] \\
& \frac{\partial F\left(Z_{-i n j}\right)}{\partial\left(Z_{-i n j}\right)}=\frac{\mu}{2 a}\left(a-F\left(Z_{-i n j}\right)\right)\left(a+F\left(Z_{-i n j}\right)\right)
\end{aligned}
$$


The set of inputs and desired outputs of NN are extracted from the PI controller of designed PI controller based MRAC scheme. A back propagation $\mathrm{NN}$ is trained till it reaches a certain fixed error. Here, the network is trained to reach an error goal of 0.0005 .

Training the Back Propagation network requires the following steps:

1. Initialize the weights and biases in the network randomly.

2. Apply inputs to the network through the input nodes.

3. Apply the target output values.

4. Calculate the error between the output and the target output.

5. Repeat steps 1-4 until the error for the entire network is acceptably low.

The structure of the NN for NN-MRAC scheme is shown in Fig. 3.

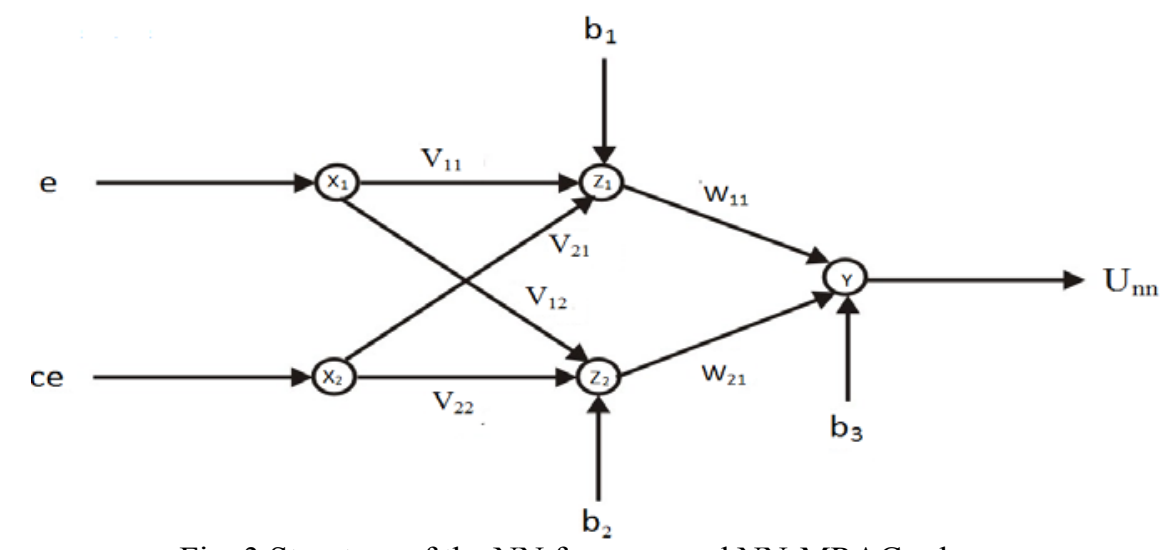

Fig. 3 Structure of the NN for proposed NN-MRAC scheme

\section{Results and Discussion}

In this section, the results of computer simulation for the conventional MRAC, PI-MRAC and NN-MRAC schemes are evaluated by applying input of varying magnitude under nonlinearities and disturbances in the plant. The same series of noise disturbance has been applied for each simulation. The results show the effectiveness of the proposed NN-MRAC scheme and reveal its performance superiority to the conventional MRAC technique. A detailed simulation comparison between the three schemes has been carried out using with two examples.

5.1. Example1. In this example, the nonlinearity component of backlash and disturbances (random noise signal) in the input of linear system are shown in Fig.4

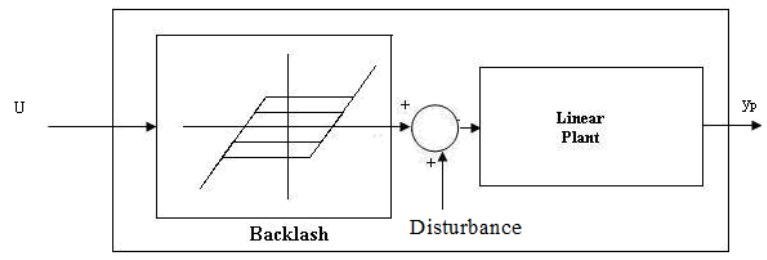

Fig. 4 Nonlinear System

The results of computer simulation are obtained by using MATLAB environment for the conventional MRAC, PI-MRAC and NN-MRAC schemes with MATLAB for time duration $\mathrm{t}=[0,50] \mathrm{s}$

A third order system with the transfer function

$G(S)=\frac{S^{2}+4 S+3.75}{S^{3}+5 S^{2}+7 S+2.5}$

is used to study and the reference model is chosen as

$G_{M}(S)=\frac{S^{2}+4 S+3.75}{S^{3}+6 S^{2}+11 S+6}$

which has relative degree $\mathrm{n}^{*}=1$

The input to the reference model is chosen as $r(t)=12$

The initial value of conventional MRAC scheme the controller parameters are chosen as $\theta(0)=[3,18,-8,3]^{\mathrm{T}}$. $U_{m r}$ is the control input of the plant for conventional MRAC scheme denoted by 
$U_{m r}=\theta^{T} \omega$

where $\theta=\left[\theta_{1}, \theta_{2}, \theta_{3}, C_{0}\right]^{T}$ is the update law vector, $\omega=\left[\omega_{1}, \omega_{2}, y_{p}, r\right]^{T}$ is the regressor vector and

$\dot{\omega}_{1}=F \omega_{1}+g u_{p}$

$\dot{\omega}_{2}=F \omega_{2}+g y_{p}$

where $F$ is an $(n-1) *(n-1)$ stable matrix such that $\operatorname{det}(S I-F)$ is a Hurwitz polynomial whose roots include the zeros of the reference model and that $(F, g)$ is a controllable pair

The PI controller gains can be selected as high as possible, but are limited by the noise. In the PI-MRAC scheme, the value of the PI controller gains $\mathrm{K}_{\mathrm{p}}=54$ and $\mathrm{K}_{\mathrm{i}}=92$, were shown to provide a better performance for the PI-MRAC scheme. The $U_{m p}$ is the control input of the plant for the PI-MRAC scheme

$U_{m p}=U_{m r}+U_{p i}$

The simulink model of the PI-MRAC scheme developed is given in Fig. 5

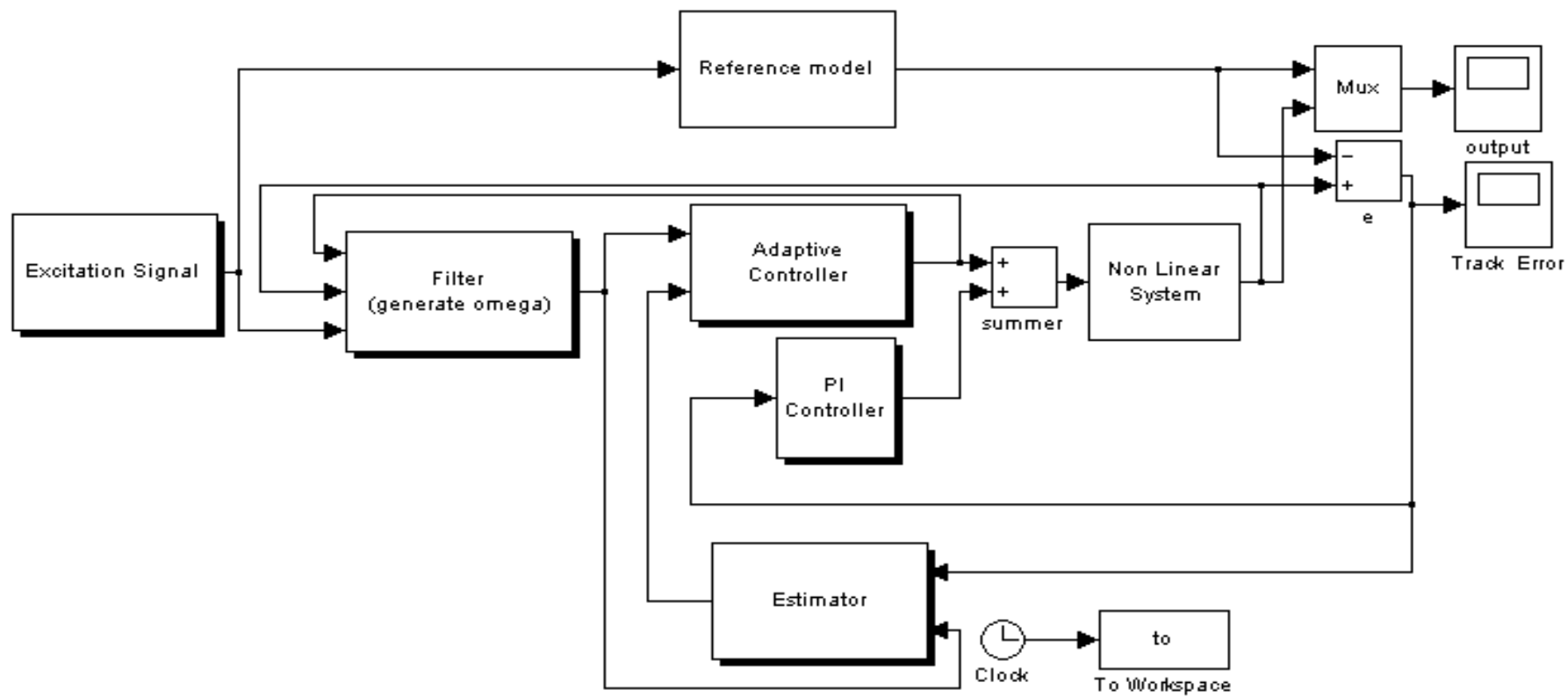

Fig.5 Simulink Model of the PI-MRAC scheme

In the NN-MRAC scheme, the details of the trained network are shown in Fig. 6

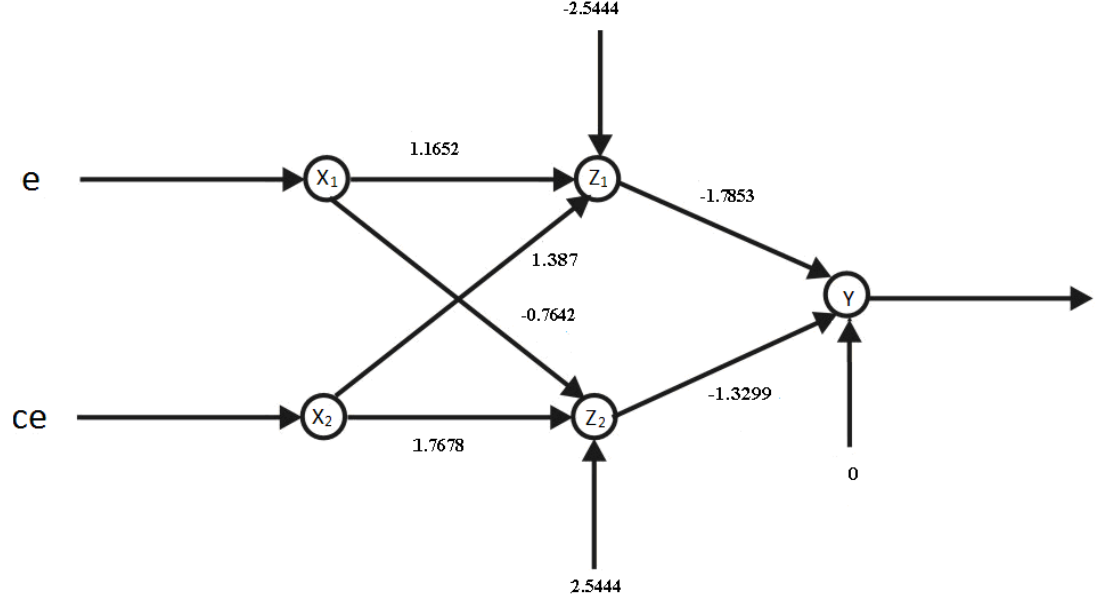

Fig.6.Details of the trained network of Example1 
The simulink model of the proposed NN-MRAC scheme developed is given in Fig .7

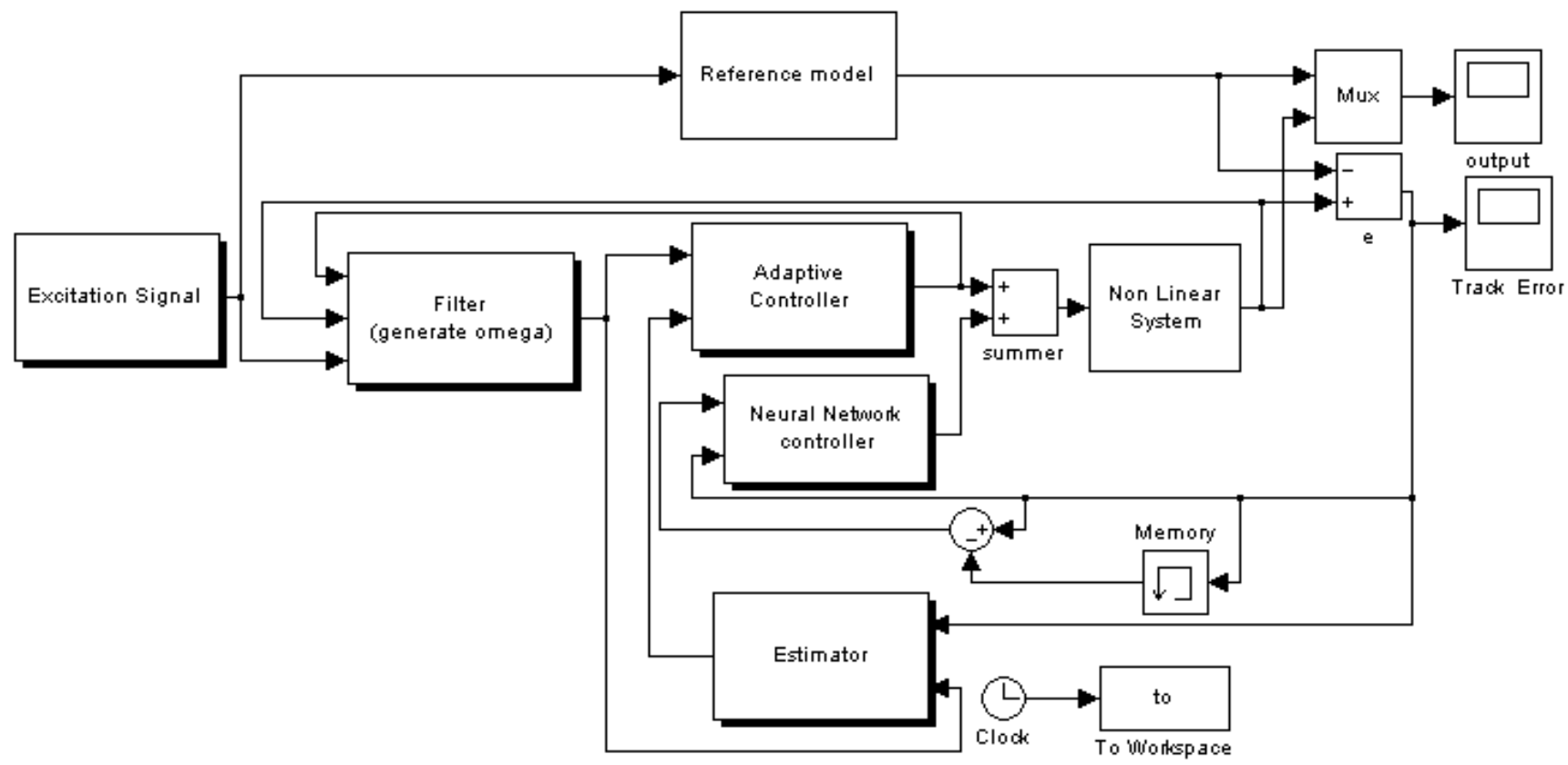

Fig.7 Simulink Model of the NN-MRAC scheme

Figs 8-10 shows the performance of the MRAC, PI-MRAC and proposed NN-MRAC schemes of Example1 with input of $r(t)=12$ under disturbance and nonlinearity component of backlash in the plant.

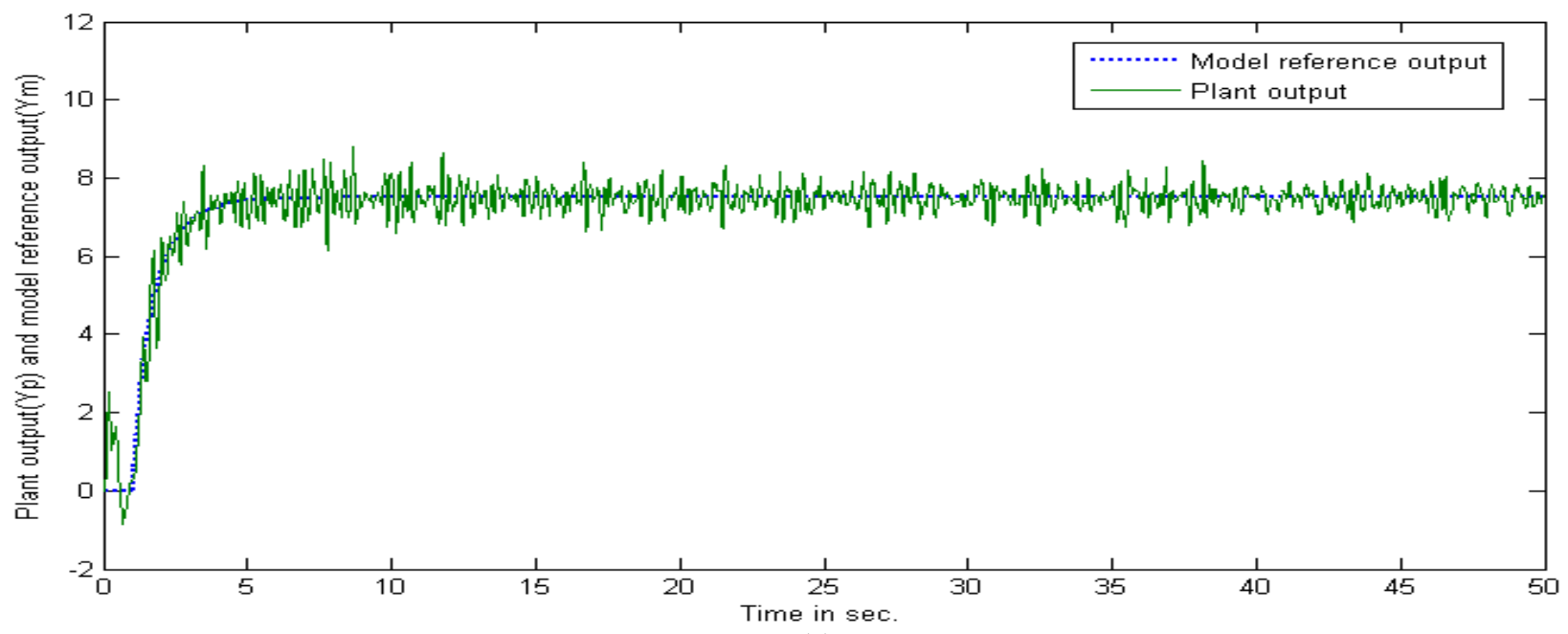

(a) 


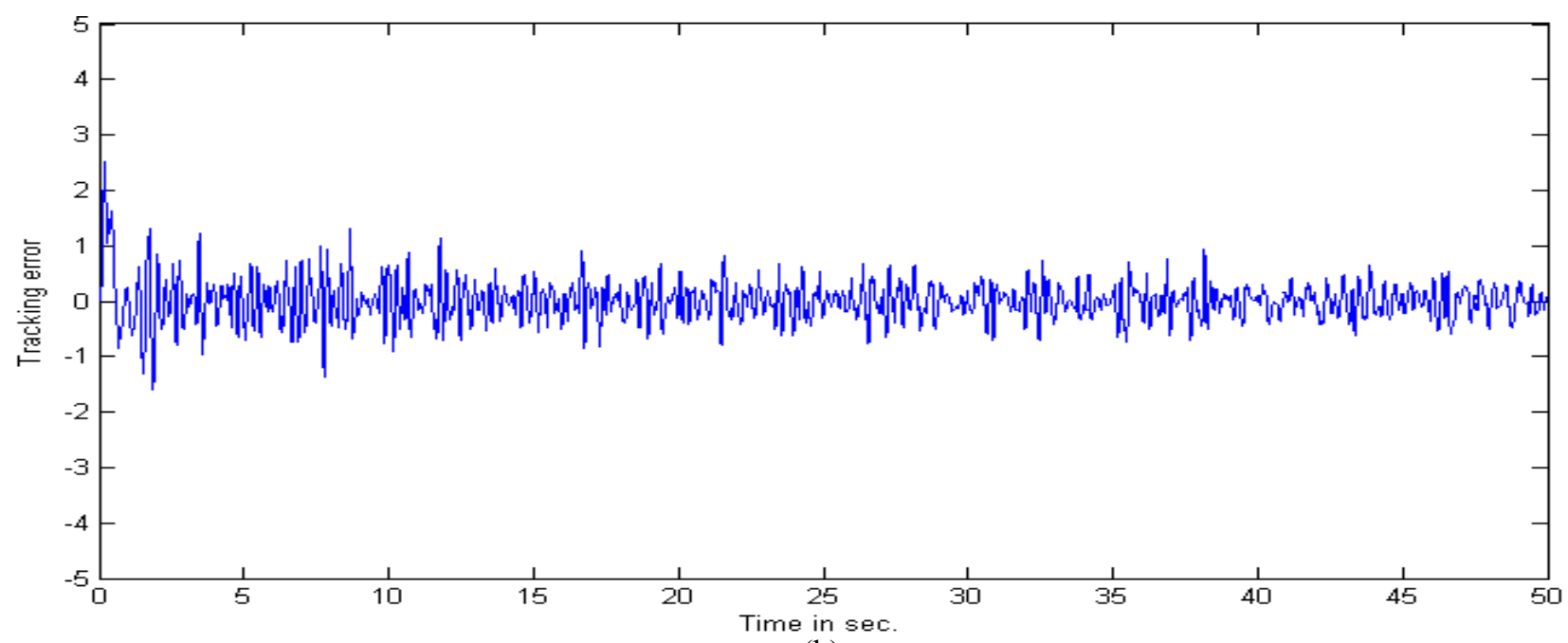

(b)

Fig. 8 Response of the conventional MRAC scheme :(a) Plant and model reference response; (b) Tracking error

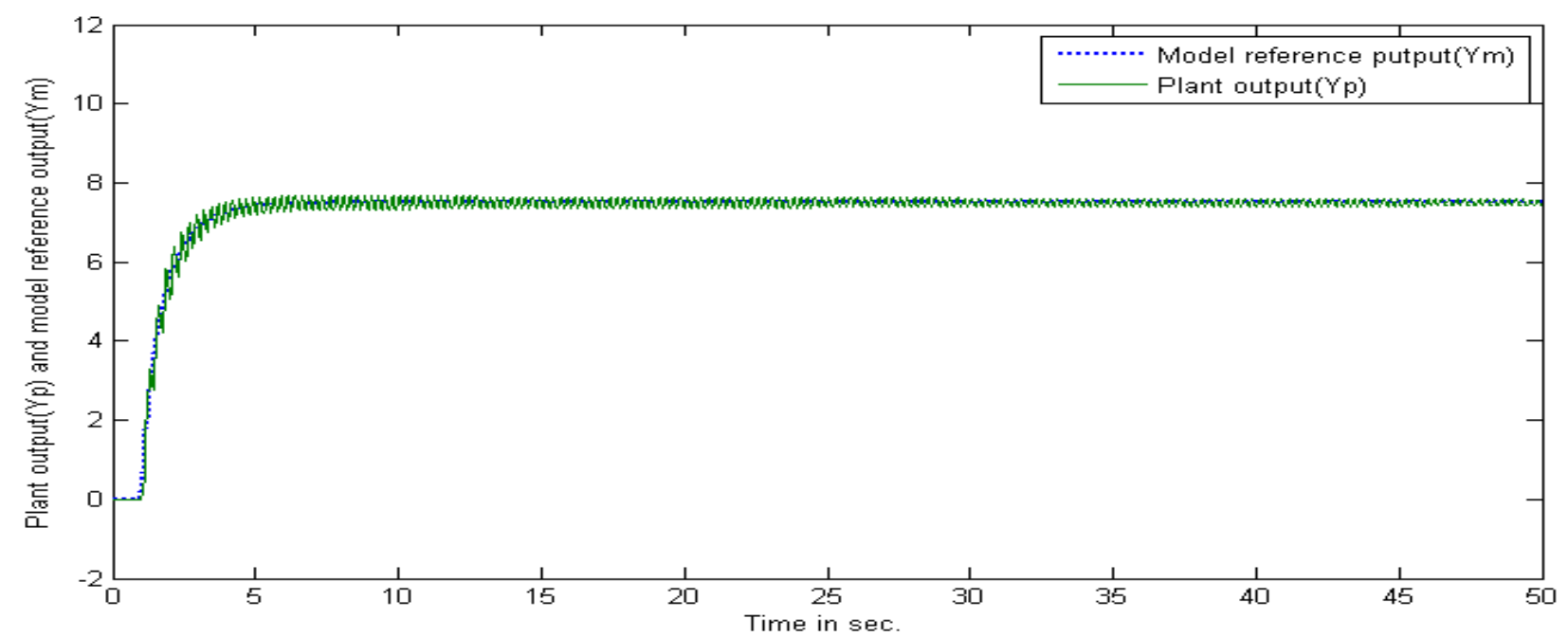

(a)

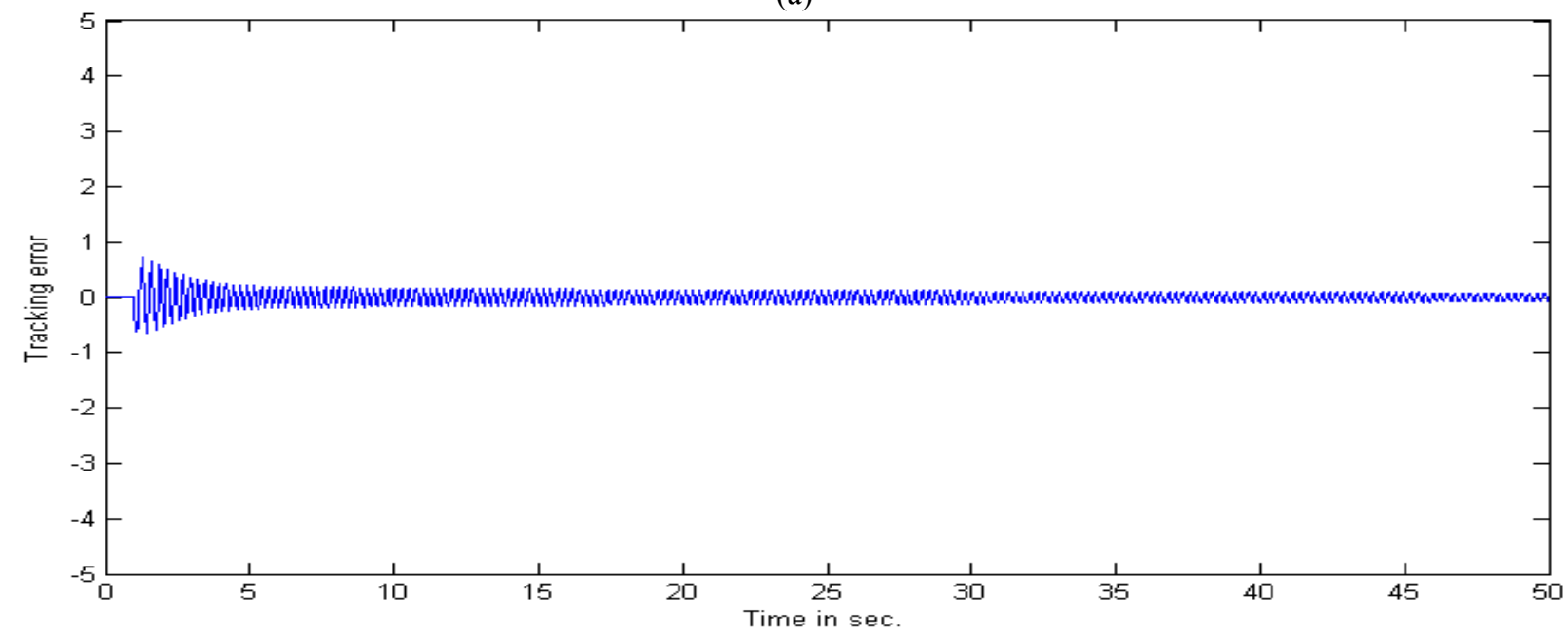

(b)

Fig. 9 Response of the PI- MRAC scheme :(a) Plant and model reference response; (b) Tracking error. 


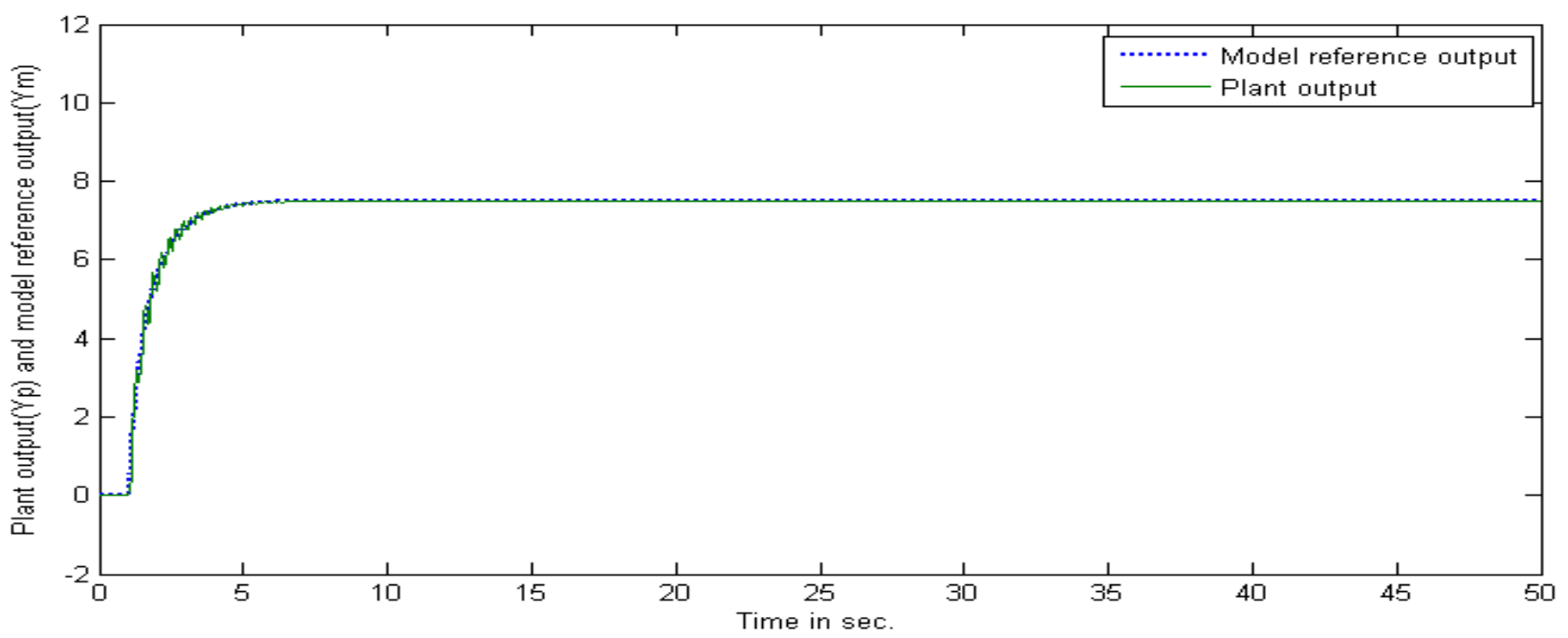

(a)

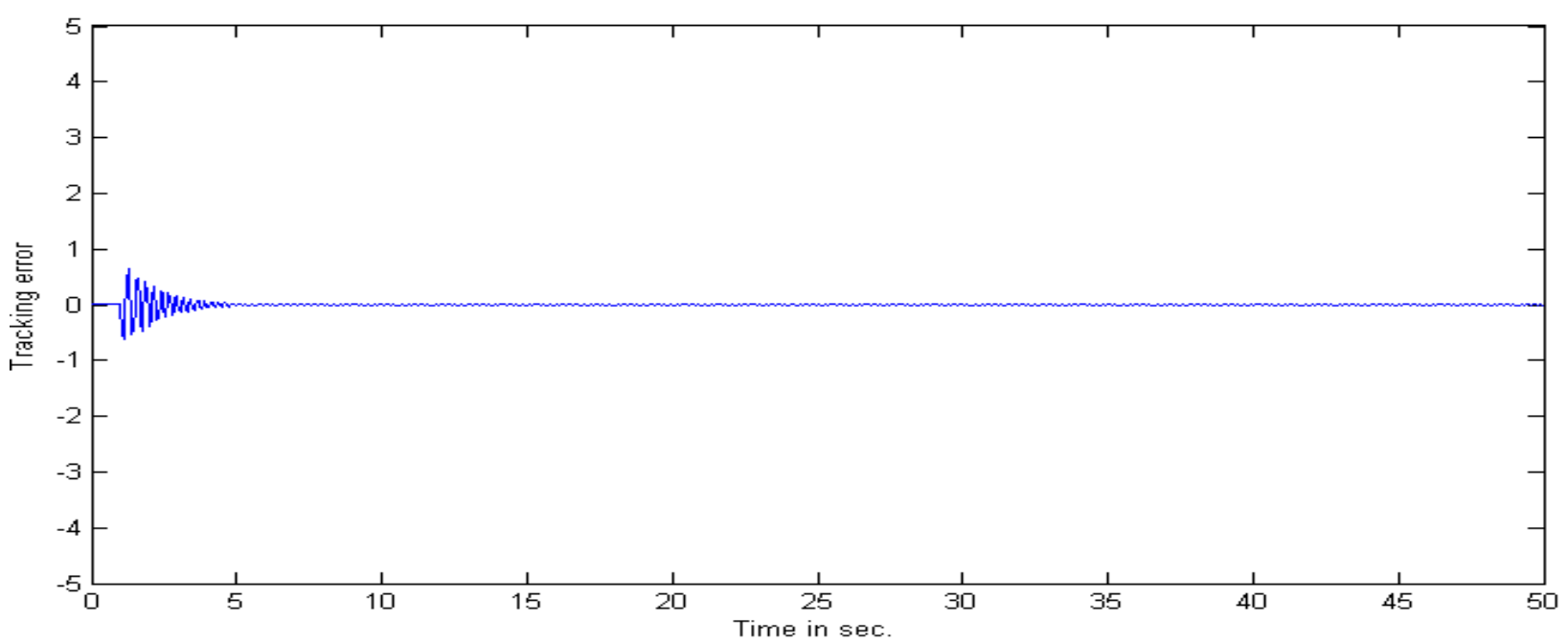

(b)

Fig. 10 Response of the NN-MRAC scheme :(a) Plant and model reference response; (b) Tracking error

5.2. Example2. In this example, the nonlinearity component of Dead zone and disturbances (random noise signal) in the input of linear system

Consider a second order system with the transfer function.

$G(S)=\frac{1}{S^{2}+3 S-10}$

is used to study and the reference model is chosen as

$G_{M}(S)=\frac{5}{S^{2}+10 S+25}$

which has relative degree $n^{*}=2$

The input to the reference model is chosen as $\mathrm{r}(\mathrm{t})=20+5 \sin 4.9 \mathrm{t}$.

The output error is $e=y_{p}-y_{m}$ and the $U_{m r}$ is the control input of the plant for conventional MRAC denoted by

$U_{m r}=\theta^{T} \omega+\dot{\theta}^{T} \Phi=\theta^{T} \omega-\theta^{T} \Gamma \phi e_{1} \operatorname{sgn}\left(K_{p} / K_{m}\right)$

where $\theta=\left[\theta_{1}, \theta_{2}, \theta_{3}, C_{0}\right]^{T}$ is the update law vector, $\omega=\left[\omega_{1}, \omega_{2}, y_{p}, r\right]^{T}$ is the regressor vector and

$\dot{\omega}_{1}=F \omega_{1}+g u_{p}$

$\dot{\omega}_{2}=F \omega_{2}+g y_{p}$

where $F$ is an $(n-1) *(n-1)$ stable matrix such that $\operatorname{det}(S I-F)$ is a Hurwitz polynomial whose roots include the zeros of the reference model and that $(F, g)$ is a controllable pair

The initial value of the conventional MRAC scheme controller parameters are chosen as $\theta(0)=[0.5,0,0,0]^{\mathrm{T}}$. 
The PI controller gains can be selected as high as possible, but are limited by the noise. In the PI-MRAC scheme, the value of the PI controller gains $\mathrm{K}_{\mathrm{p}}=8$ and $\mathrm{K}_{\mathrm{i}}=92$, were shown to provide an optimal performance for the PI-MRAC scheme. The $U_{m p}$ is the control input of the plant for the PI-MRAC scheme

$U_{m p}=U_{m r}+U_{p i}$

The simulink model of the proposed NN-MRAC scheme developed is given in Fig 11

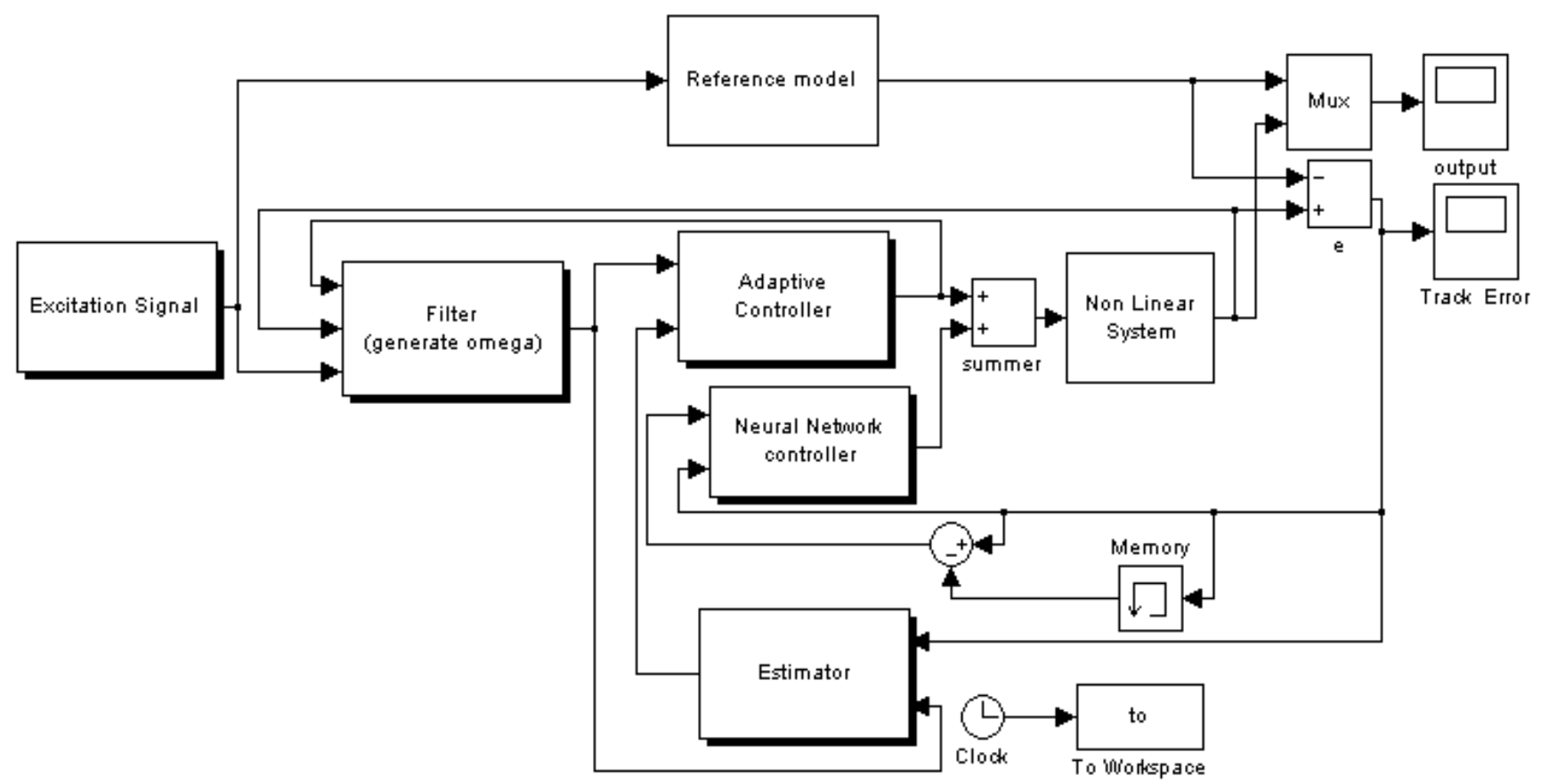

Fig. 11 Simulink Model of the proposed NN-MRAC scheme

The $U_{m n}$ is the control input of the plant for the NN-MRAC scheme.

$$
U_{m n}=U_{m r}+U_{n n}
$$

In the proposed NN-MRAC scheme, the details of the trained network are shown in Fig. 12.

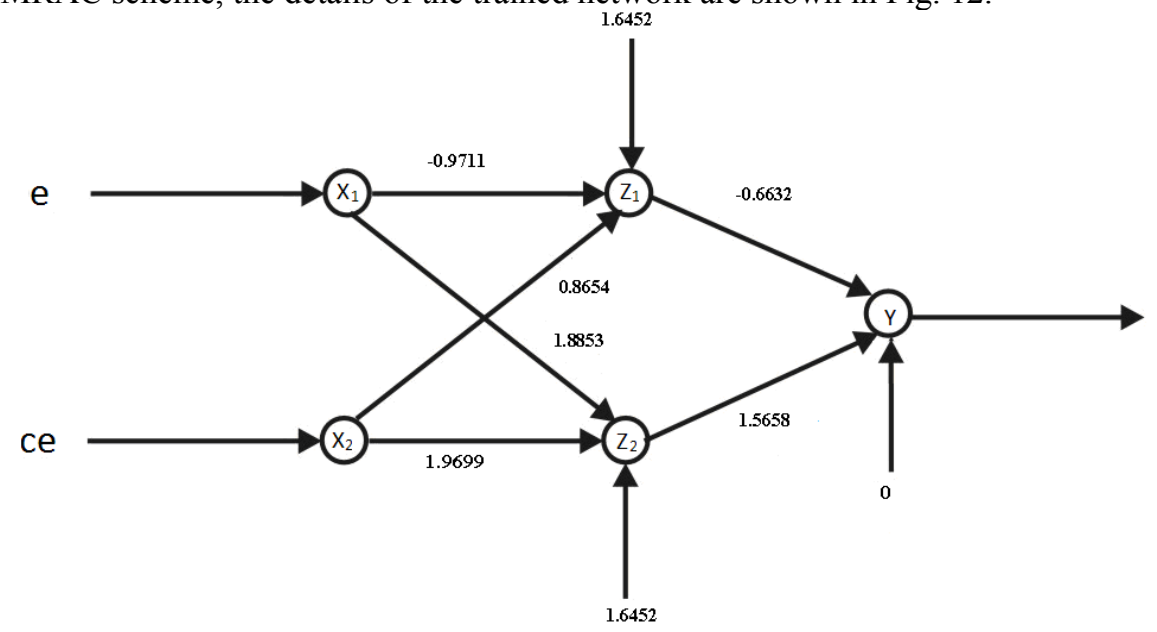

Fig. 12 Details of the trained network of Example2

Figs 13-15 shows the performance of the MRAC, PI-MRAC and proposed NN-MRAC schemes of Example2 with input of $r(t)$ $=20+5 \sin 4.9 t$ under disturbance and nonlinearity component of Dead zone in the plant. 

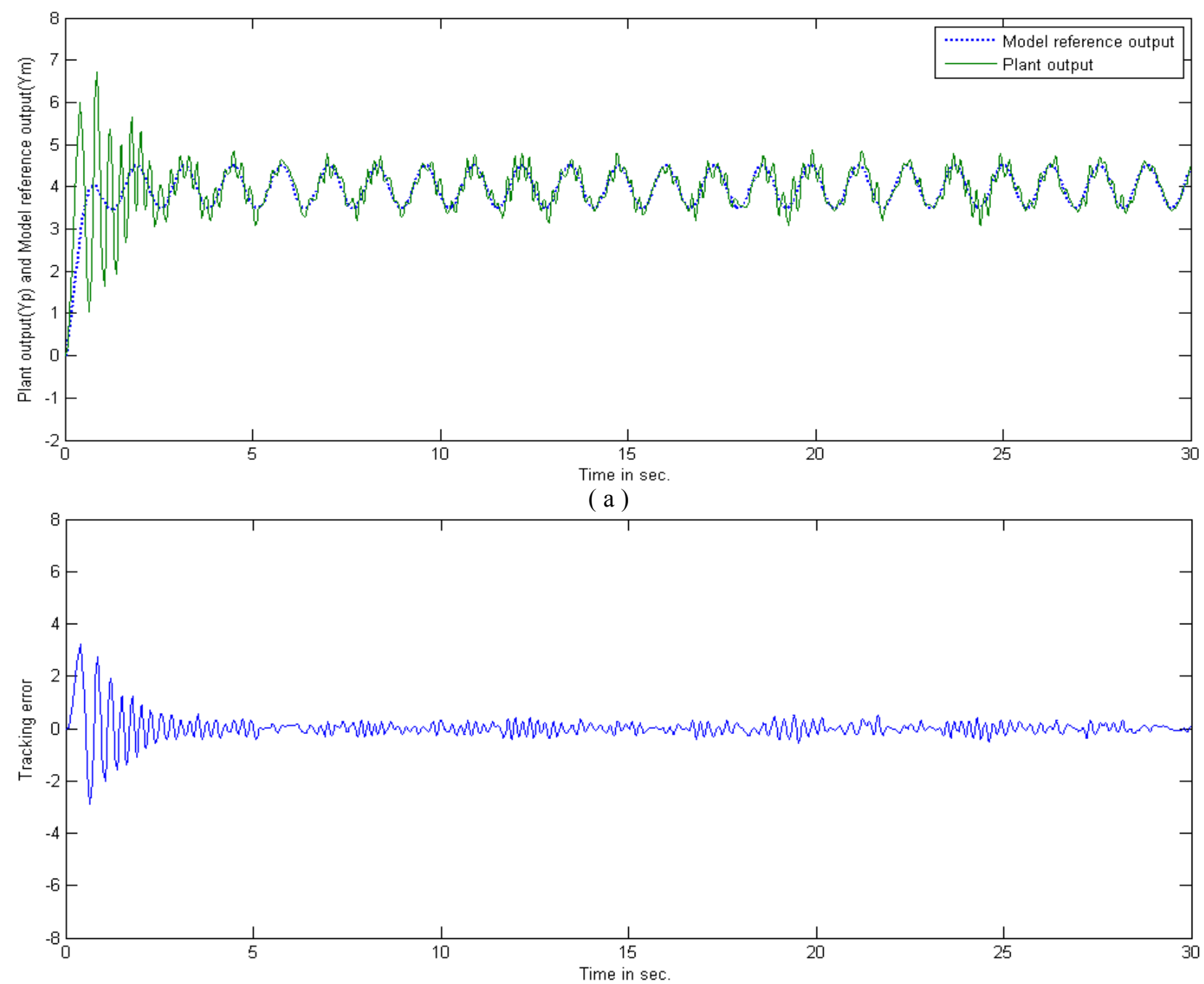

(b)

Fig. 13 Response of the conventional MRAC scheme :(a) Plant and model reference response; (b) Tracking error

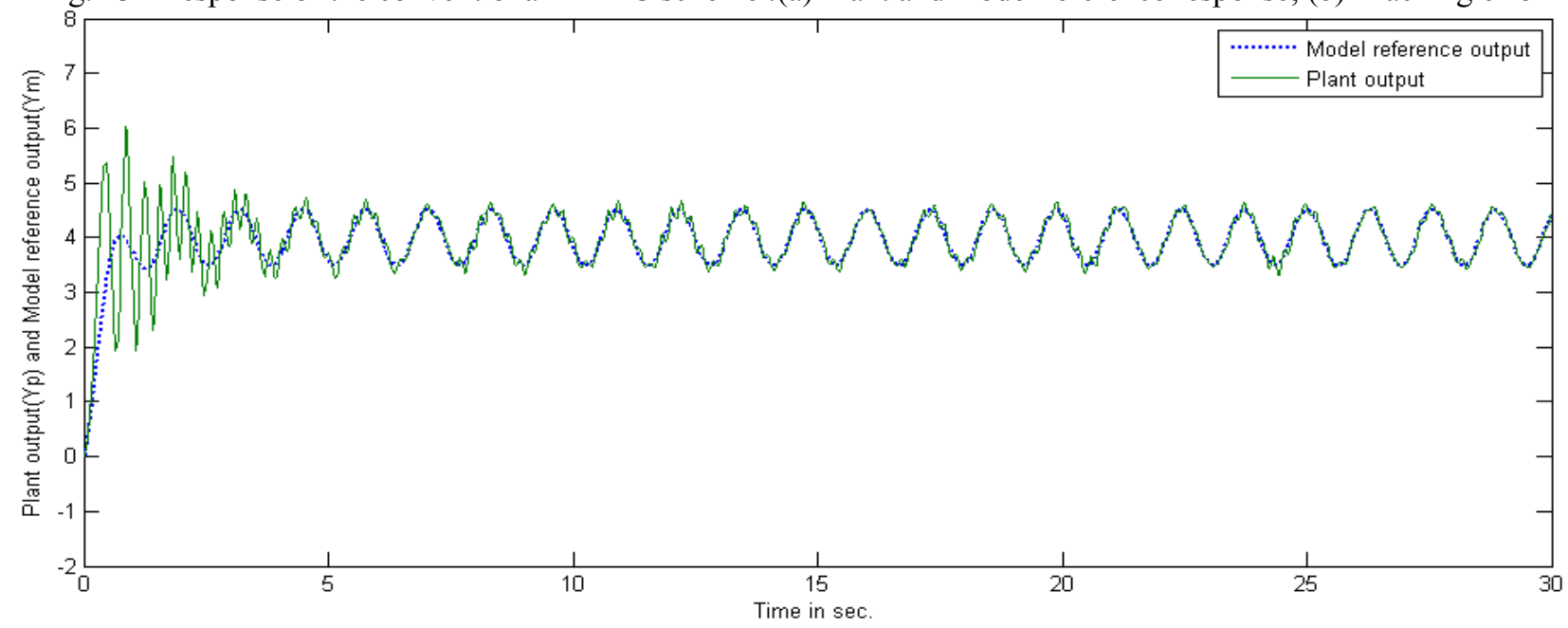

(a) 


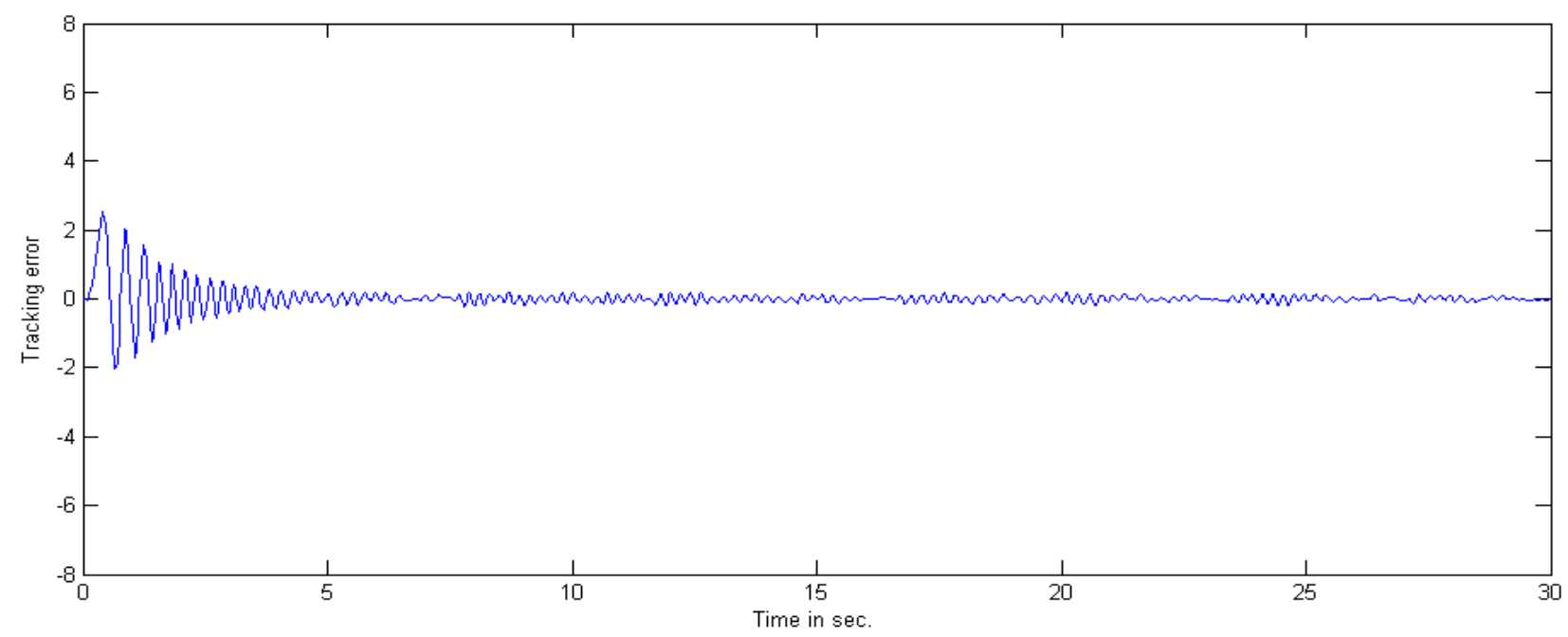

(b)

Fig. 14 Response of the PI-MRAC scheme :( a) Plant and model reference response; (b) Tracking error

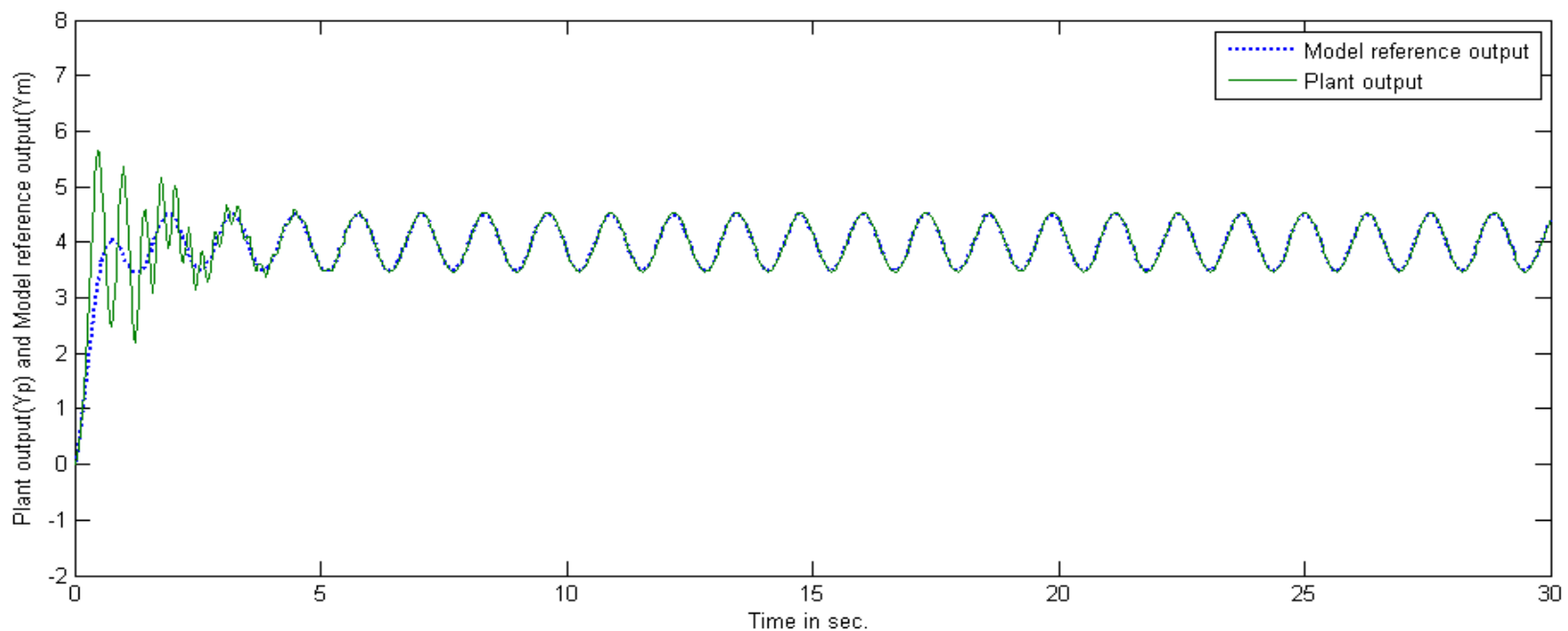

(a)

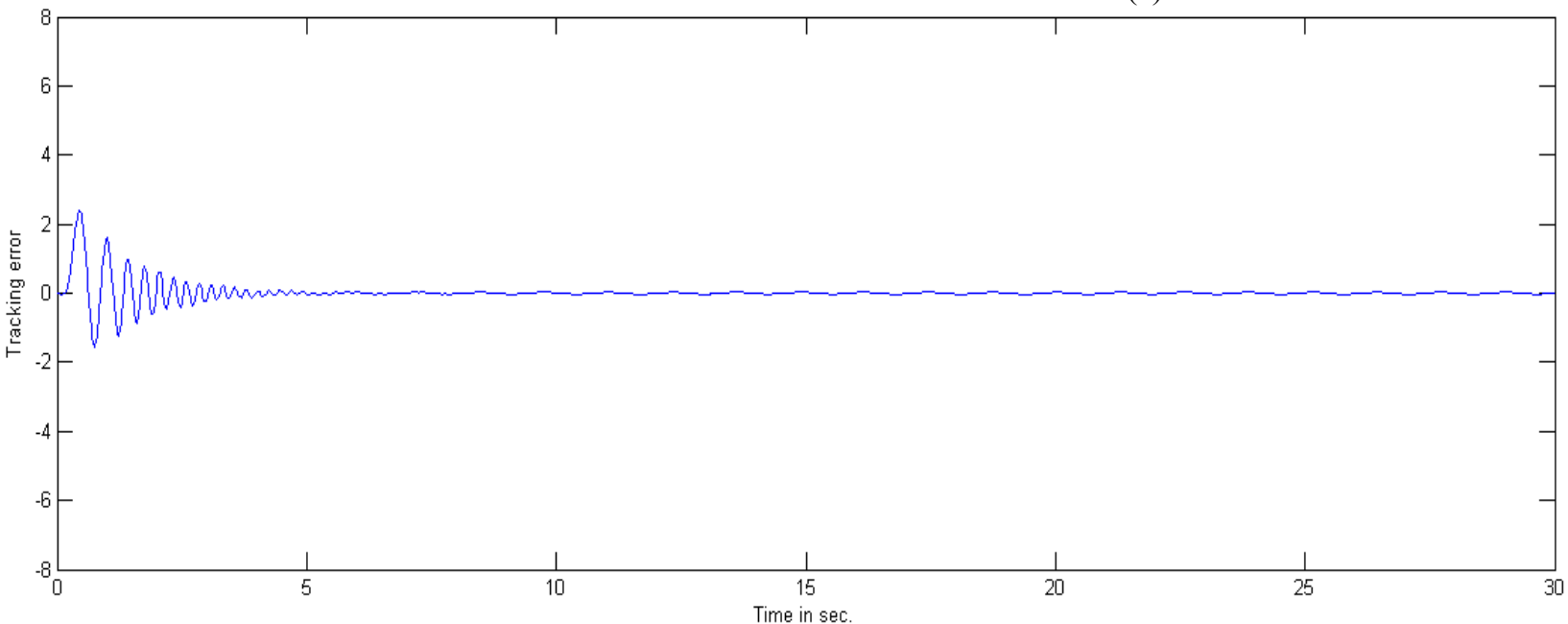

(b)

Fig. 15 Response of the NN-MRAC scheme :(a) Plant and model reference response; (b) Tracking error

The performance of the conventional MRAC, PI-MRAC and NN-MRAC scheme is evaluated by applying inputs of varying magnitude under nonlinearities and disturbance in the plant. The results show the effectiveness of the NN-MRAC scheme to force the plant to follow the model, under uncertainties. Extensive simulation tests were carried out to compare the three adaptation 
schemes: conventional MRAC, PI-MRAC scheme and NN-MRAC scheme. In the simulation results of conventional MRAC, PIMRAC and NN-MRAC schemes, the dotted line and solid line represents the model reference trajectory and plant trajectory respectively. In conventional MRAC scheme, the plant output is poor with large overshoots and oscillations as shown in Figs. 8 and 13.

Figs. 9 and 14 show the response of the PI-MRAC scheme. In this case, the overshoots and the oscillations are much smaller, yielding a much better performance than the conventional MRAC scheme. However, due to the continuous variation in the system parameters and the operating conditions, in addition to the nonlinearities present in the system, PI-MRAC scheme may not be able to provide the required performance. In the proposed NN-based MRAC scheme, the plant output has tracked with the reference model output and the tracking error becomes zero within 6 seconds with less control effort as shown in Figs.10 and 15 . The responses performed by the control algorithm only with the model reference adaptive controller are observed to be inferior to that of the control algorithm both with NN controller and the model reference adaptive controller. Also, the response of the control algorithm only with the model reference adaptive controller shows large overshoot and oscillation. Further, the response of the output performed by the control algorithm both with the NN controller and the model reference adaptive controller shows more satisfactory results for the bounded disturbances with unknown as well as time-varying characteristics than that of the control algorithm only with the model reference adaptive controller. From the simulation results, because of the existing nonlinearities and bounded disturbances, the controlled system using the control algorithm only using the model reference adaptive controller will be unstable. But when using the $\mathrm{NN}$ and the model reference adaptive controller in coordination in which the control law is used to cope with nonlinearities and bounded disturbances, the controlled system can be robustly stabilized all the time. From the above discussions, the proposed control algorithm both with the neural network and the conventional model reference adaptive controller can be a promising way to tackle the problem of controlling the nonlinear systems and bounded time-varying disturbances.

From the above simulations, it is shown that the control algorithm using only the model reference adaptive controller will not stabilize the nonlinear controlled systems with disturbances. From Figs. 10 and 15, it is seen that the control algorithm both with the neural network control and the model reference adaptive controller working in coordination can cope up with the uncertain dynamic system and bounded disturbances, but the control algorithm without the neural network compensating control cannot. Compared to the conventional MRAC, PI-MRAC scheme and NN-MRAC scheme still show a faster response during transients. Moreover, the NN-MRAC scheme shows faster response compared to the PI-MRAC scheme. An optimal response was obtained for the NN-MRAC scheme compared to the PI MRAC scheme. The proposed NN- MRAC scheme shows better control results compared to those by the conventional MRAC and PI-MRAC scheme.

From these simulation results it is observe that:

1. In conventional MRAC the plant output is not tracked with the reference model output. The conventional MRAC fails completely under the action of the external disturbance and nonlinearities, where a degradation in the performance due to overshoot is observed.

2. The PI-MRAC improves the transient and steady state performances compared with the conventional MRAC. In the PIMRAC scheme, the plant output is nearly track with the reference model output. However it will not be able to provide the required performance.

3. The proposed NN-MRAC design approach can keep the plant output in track with the reference model and tracking error becomes zero within 6 seconds. The proposed NN-MRAC controller gives better performances in terms of steady-state error, settling time and overshoot. Hence it can be concluded that the proposed NN-MRAC scheme is more robust performance than the classical MRAC and PI-MRAC scheme.

On the contrary, the proposed method has much less error than the conventional method in spite of nonlinearities and disturbance. The simulation results have confirmed the efficiency of the proposed NN-MRAC scheme for applying disturbances and nonlinearities.

5.3. Implementation issue. The proposed method can be widely used in most of the industrial nonlinear and complex applications such as machine tools, industrial robot control, position control, and other engineering practices. The proposed NNMRAC is relatively simple and does not require complex mathematical operations. It can be readily implemented using conventional rnicroprocessors or microcontrollers. The execution speed of the NN-MRAC scheme can be improved by using advanced processors such as Reduced Instruction Set Computing (RISC) processors or Digital Signal Processors (DSP's) or ASIC's (Application Specific Integrated Circuits).

\section{Conclusion}

In this paper, a NN-MRAC scheme is proposed to replace the PI controller of PI based MRAC by a neural network. In NN MRAC the training patterns of neural network are extracted from the PI controller of designed PI -MRAC scheme. A detailed simulation comparison between the three schemes has been carried out using with two examples. The proposed NN-MRAC controller shows very good tracking results when compared to the conventional MRAC and the PI-MRAC system. Simulations and analyses have shown that the transient performance can be substantially improved by proposed NN-MRAC scheme In proposed NN-MRAC scheme, the system output tracks very closely the reference model in spite of the disturbances and 
nonlinearities. Thus the NN-MRAC controller is found to be extremely effective, efficient and useful. However, the application of the new adaptation schemes does not considerably improve the steady-state performance. Development of a neurofuzzy networkbased MRAC scheme is recommended as the future works for improve the steady state performance of the system with the presence of disturbances and nonlinearities. Due to its simple operation, the proposed NN-MRAC can be readily implemented using conventional microprocessors.

\section{References}

Astrom K.J. and Wittenmark B.. 1995. Adaptive control (2nd Ed.) Addison-Wesley.

Chen. S, Billings, S.A and Grant, P.M.,1991, Nonlinear system identification using neural network, Int. J. Control, Vol. 51, pp. $1191-1214$

Chen, F.C, 1990, "Back propagation neural networks for non linear Self - tuning adaptive control”, IEEE contr. Syst. Mag., 10,40-48.

Arciniegas J.I., A.H. Eltimashy and K.J. Cios, 1997. Neural networks based adaptive control of flexible arms, Neurocomputing, Vol.17, No. 314, pp.141-157.

Ahmed M.S. 2000. Neural net-based direct adaptive controller a class of nonlinear plants, IEEE Trans Autom. Control. Vol.45, No, 1, pp. 119-123.

Biyanto T.R., Bambang L. Widjiantoro, Ayman Abo Jabal, Titik Budiati 2010. Artificial neural network based modeling and controlling of distillation column system, International Journal of Engineering, Science and Technology, Vol. 2, No. 6, pp. 177-188.

Calise A.J., N. Hovakimyan and M. Idan, 2001. Adaptive output feedback control of nonlinear system using neural networks, Automatica, Vol. 37, No.8, pp. 1201-1211.

Cho, H.C.; Fadali, M.S.; Lee, K.S.; Kim, N.H., 2009. Model reference adaptive control-based adaptive current control scheme of a PM synchronous motor with an improved servo performance”, IET on Electric Power Applications ,Vol.3, No.1. pp. 8 - 18.

Fadali, M.S.; Lee, K.S.; Kim, N.H., 2010. Adaptive position and trajectory control of autonomous mobile robot systems with random friction, IET on Control Theory \& Applications, Vol.4, No.12. pp. 2733-2742.

Guchi,Y and Sakai, H, 1991, A nonlinear regulator design in the presence of system uncertainities using multilayered neural network, IEEE Trans. Neural Network, Vol. 2, pp. 427-432.

Huh, S.-H and Bien, Z. 2007. Robust sliding mode control of a robot manipulator based on variable structure-model reference adaptive control approach, IET on Control Theory \& Applications, Vol.1, No.5. pp. 1355-1363, Oct. 2007

Kawalo, M. Furukawa.K and Suzuki, R., 1987, A hierarchical neural network for control and learning of voluntary movement, Biol Cybern, Vol. 57, pp. 169-185.

Kamalsudan S. and Ghandakly A.A. 2010. A neural network parallel adaptive controller for fighter aircraft pitch- rate tracking, IEEE Transaction on Instrumentation and Measurement

Kamalasadan S., A. Ghandakly, 2007. A neural network parallel adaptive controller for dynamic system control, IEEE Transactions on Instrumentation and Measurement, Vol.56, No.5. pp. 1786-1796.

Loannou P.A., Sun J., 1996. Robust Adaptive control, Upper Saddle River, NJ: Prentice-Hall.

Liu, C.C. and Chen, F.C., 1993, Adaptive control of non - linear continuous - time systems using neural networks- general relative degree and MIMO cases, Int. J. Control, Vol. 58, pp. 317-335.

Mirkin, B.; Gutman, P.-O., 2010. Robust adaptive output-feedback tracking for a class of nonlinear time-delayed plants, IEEE Transactions on Automatic Control, Vol.55, No.10, pp. 2418-2424.

Mofal M.B. and Calise A.J. 1997. Robust adaptive control of uncertain nonlinear systems using neural network", in Proc. Amer Control Cont .pp. 1996-2000.

Narendra, K.S and parthasarathy, 1990, "identification and control of dynamic systems using neural network". IEEE Tans. Neural network 1, 4-27

Sadighi,A. Kim, W.“ Adaptive-Neuro-Fuzzy-Based Sensorless Control of a Smart-Material Actuator”, IEEE/ASME Transactions on Mechatronics, vol.1, no.2, pp. 371 - 379, Jan. 2011

Sanner R.M. and J,E. Slotine, "Gaussian Networks for Direct Adaptive Control", IEEE trans neural networks vol3, no.6. pp.837763, Nov1992

Vas P., Sensorless Vector and Direct Torque Control. New York: Oxford Univ. Press, 1998.

Xiang-Jie Liu; F. Lara-Rosano; C.W. Chan, 2004. Model-reference adaptive control based on neurofuzzy networks, IEEE Transactions on Systems, Man, and Cybernetics, Part C: Applications and Reviews, Vol.34, No.3. pp. 302-309.

Xiaofang Y.; Yaonan W.; Wei S.; Lianghong W., 2010. RBF networks-based adaptive inverse model control system for electronic throttle, IEEE Transactions on Control Systems, Vol. 18, No. 3. pp. 750 - 756.

Yamada, T and Yabuta,T., 1992,Neural network controller using autotunning method for non linear function. IEEE Trans. Neural Network, Vol. 3, pp. 595-601.

Zhai Y., Nounou1 M., Nounou H. and Al-Hamidi Y. 2010. Model predictive control of a 3-DOF helicopter system using successive linearization, International Journal of Engineering, Science and Technology,.Vol. 2, No. 10, pp. 9-19. 


\section{Biographical notes}

R.Prakash received his B.E degree from Government College of Technology, affiliated to Bharathiyar University, Coimbatore, Tamilnadu, India in 2000 and completed his M.Tech degree from the College of Engineering, Thiruvanandapuram, Kerala, India, in 2003. He is currently working for his doctoral degree at Anna University, Chennai, India. He has been a member of the faculty Centre for Advanced Research, Muthayammal Engineering College, Rasipuram, Tamilnadu, India since 2008. His research interests include Adaptive Control, Fuzzy Logic and Neural Network applications to Control Systems.

R.Anita received her B.E Degree from Government College of Technology in 1984 and completed her M.E Degree from Coimbatore Institute of Technology, Coimbatore, India in 1990, both in Electrical and Electronics Engineering. She obtained her Ph.D degree in Electrical and Electronics Engineering from Anna University, Chennai, India, in 2004. At present she is working as Professor and Head of Department of Electrical and Electronics Engineering, Institute of Road and Transport Technology, Erode, India. She has authored over sixty five research papers in International, National journals and conferences. Her areas of interest are Advanced Control Systems, Drives and Control and Power Quality

Received December 2010

Accepted April 2011

Final acceptance in revised form July 2011 